\title{
Transcriptomic comparison of the self- pollinated and cross-pollinated flowers of Erigeron breviscapus to analyze candidate self-incompatibility-associated genes
}

Wei Zhang ${ }^{1,2+}$, Xiang Wei ${ }^{2+}$, Heng-Lin Meng ${ }^{2}$, Chun-Hua Ma ${ }^{1}$, Ni-Hao Jiang ${ }^{3}$, Guang-Hui Zhang ${ }^{1 *}$ and Sheng-Chao Yang ${ }^{1 *}$

\begin{abstract}
Background: Self-incompatibility (SI) is a widespread and important mating system that promotes outcrossing in plants. Erigeron breviscapus, a medicinal herb used widely in traditional Chinese medicine, is a self-incompatible species of Asteraceae. However, the genetic characteristics of SI responses in E. breviscapus remain largely unknown. To understand the possible mechanisms of $E$. breviscapus in response to $\mathrm{Sl}$, we performed a comparative transcriptomic analysis with capitulum of E. breviscapus after self- and cross-pollination, which may provide valuable information for analyzing the candidate SI-associated genes of E. breviscapus.

Methods: Using a high-throughput next-generation sequencing (Illumina) approach, the transcriptionexpression profiling of the different genes of $E$. breviscapus were obtained, some results were verified by quantitative real time PCR (qRT-PCR).

Results: After assembly, 63,485 gene models were obtained (average gene size 882 bp; N50 = 1485 bp), among which 38,540 unigenes ( $60.70 \%$ of total gene models) were annotated by comparisons with four public databases ( $\mathrm{Nr}$, SwissProt, KEGG and COG): 38,338 unigenes (60.38 \% of total gene models) showed high homology with sequences in the $\mathrm{Nr}$ database. Differentially expressed genes were identified among the three cDNA libraries (non-, self- and crosspollinated capitulum of E. breviscapus), and approximately 230 genes might be associated with SI responses. Several these genes were upregulated in self-pollinated capitulum but downregulated in cross-pollinated capitulum, such as SRLK (SRK-like) and its downstream signal factor, MLPK. qRT-PCR confirmed that the expression patterns of EbSRLK1 and EbSRLK3 genes were not closely related to SI of E. breviscapus.

Conclusions: This work represents the first large-scale analysis of gene expression in the self-pollinated and crosspollinated flowers of E. breviscapus. A larger number of notable genes potentially involved in SI responses showed differential expression, including genes playing crucial roles in cell-cell communication, signal transduction and the pollination process. We thus hypothesized that those genes showing differential expression and encoding critical regulators of SI responses, such as MLPK, ARC1, CaM, EXO70A1, MAP, SF21 and Nod, might affect SI responses in E. breviscapus. Taken together, our study provides a pool of SI-related genes in E. breviscapus and offers a valuable resource for elucidating the mechanisms of $\mathrm{SI}$ in Asteraceae.
\end{abstract}

Keywords: Erigeron breviscapus, Transcriptome, Self-incompatibility, Digital gene expression

\footnotetext{
*Correspondence: zgh73107310@163.com; shengchaoyang@163.com

${ }^{\dagger}$ Equal contributors

'Yunnan Research Center on Good Agricultural Practice for Dominant

Chinese Medicinal Materials, Yunnan Agricultural University, Kunming 650201,

Yunnan, People's Republic of China

Full list of author information is available at the end of the article
}

\section{Biomed Central}

(c) 2015 Zhang et al. Open Access This article is distributed under the terms of the Creative Commons Attribution 4.0 International License (http://creativecommons.org/licenses/by/4.0/), which permits unrestricted use, distribution, and reproduction in any medium, provided you give appropriate credit to the original author(s) and the source, provide a link to the Creative Commons license, and indicate if changes were made. The Creative Commons Public Domain Dedication waiver (http://creativecommons.org/publicdomain/zero/1.0/) applies to the data made available in this article, unless otherwise stated. 


\section{Background}

Self-incompatibility (SI) is the most widespread and important mating system that promotes outcrossing while prevents inbreeding. Many flowering plants have SI system [1], which can be classified into two types: gametophytic SI (GSI) and sporophytic SI (SSI). The incompatibility phenotype of pollen is determined by its own haploid genotype in GSI, whereas it is determined by the diploid genotype of the parent plants in SSI [2]. The Brassicaceae is considered as a hotspot of SSI, which uses the pistil-expressed receptor kinase to recognize self/non-self-pollen [3] and is controlled by a multi-allelic $S$ locus [4, 5]. $S$ alleles have high amino acid sequence divergence within species [6-9]. $S$-locus receptor kinase $(S R K)$ was identified as the female determinant [10]. S-locus cysteine-rich $(S C R)$ proteins were identified as the male determinants in the Brassicaceae [11, 12]. Binding of SCR to SRK induces autophosphorylation of SRK, which triggers a signaling cascade leading to the SI response. Arm repeat containing 1 (ARC1) and $M$-locus protein kinase $(M L P K)$ are two signaling molecules that positively mediate signal transduction. $A R C 1$ is expressed in the stigma and interacts with $S R K$ through its cytoplasmic domain [13, 14]. MLPK was identified in a recessive mutant of Brassica rapa var. Yellow Sarson. Mutation of the gene leads to SI in B. rapa Yellow Sarson [15]. Other components associated with the SI signaling pathway include thioredoxin- $h 1$ (THL1) and thioredoxin-h 2 (THL2). Moreover, $\mathrm{Ca}^{2+}$ is also involved in signal transduction of SI. In Citrus clementine, several novel genes that potentially regulate $\mathrm{Ca}^{2+}$ homeostasis were identified during self-pollen recognition [16].

Genetic studies of SI in the Asteraceae started with species such as Crepis foetida, Parthenium argentium and Cosmos bipinnatus in the 1950s. Over $60 \%$ of species in the Asteraceae are estimated to use SSI for genetic determination. First studies in these plants identified the SSI system in the Asteraceae and showed that the SI is controlled by the $S$ locus $[17,18]$. However, the precise number of $S R K$ genes required for pollen specificity and the male and female determinants underlying SSI in the Asteraceae remains unknown. Senecio squalidus (Oxford Ragwort) has been used as a model plant to study the molecular mechanism of SI in the Asteraceae. Early studies on stigma surfaces in the Asteraceae indicated that the Asteraceae species have dry type stigmas. Later, Elleman et al. performed a comparative study of pollen-stigma interactions among five different plants and showed that stigmas of Asteraceae species produce a small amount of surface secretion and are not entirely dry [19]. This finding was subsequently confirmed by Hiscock et al. in S. squalidus and other Asteraceae species, which led to a reclassification of the Asteraceae stigma as 'semi-dry' [20]. It has been concluded that SSI in Senecio species operates through a different molecular mechanism with that in Brassica [21]. However, the S. squalidus dataset shared a greater number of homologous genes with the dry stigma species than the wet stigma species [22]. The number of $S$ alleles in $S$. squalidus is low compared with other species that use SSI [23-25]. This inference was further analyzed through different transcripts of $S$. squalidus and SSH was successfully used to isolate pistil-enriched transcripts from three different S-genotypes of S. squalidus. 115 different candidates for pistil-specific genes in S. squalidus were identified [26]. Several new genes, such as membrane associated protein (MAP), sunflower-21 (SF21) and Nodulin/ $m$ tn3 gene (Nod), might be linked with SI in S.squalidus. The Senecio pistil-specific MAP was found to be expressed in the papillar cells and transmitting tissue of the stigma [22, 27]. In S. squalidus, the nucleotide sequence of MAP exhibits relatively high $S$-genotypic polymorphism, which is elevated in the extracellular region [22, 27]. The SF21 gene family was also isolated from $S$. squalidus. There are differences in these gene copies and expressed patterns [26]. Nod is expressed exclusively in the papillar cells of the $S$. squalidus stigma, where it appears to be developmentally regulated, reaching maximal expression as the stigmatic lobes reflex to expose the papillar cells [22, 27].

Erigeron breviscapus (Vant.) Hand.-Mazz, an important Chinese traditional medicinal plant $[28,29]$, is a selfincompatible species of Asteraceae. It has been used to treat cerebrovascular and cardiovascular problems [30]. Recently, accumulating studies on E. breviscapus have been focused on chemical components [31,32], pharmacological activities [32-34], and germplasm resources [35-39]. However, little is known about the genetic mechanisms of SI responses in E. breviscapus.RNA sequencing is a powerful tool to investigate the molecular biology of many angiosperm and it has been used successfully for SI in lemon and Leymus chinensis [40, 41]. To uncover critical genes associated with SI responses, we compared the gene expression profiles in capitulum of E. breviscapus during non-, selfand cross-pollination, using transcriptome sequencing and de novo assembly. After analysis, a larger number of potential SI candidate genes showing differential expression were uncovered, including genes functioning in cell-cell communication, signal transduction and the pollination process, such as MLPK, ARC1, CaM, Exo70A1, MAP, SF21 and Nod. Our study thus provides a pool of SI-related genes in E. breviscapus and offers a valuable resource to investigate the molecular mechanisms of SI responses in Asteraceae.

\section{Results}

Sequencing and de novo assembly of E. breviscapus transcriptome

Three pools (self-pollinated, cross-pollinated and nonpollinated) capitulum of mRNA samples were used to 
build libraries for high-throughput sequencing using Illumina sequencing technology. The Illumina HiSeq 2000 next-generation sequencing generated $68.68 \mathrm{M}$ raw reads comprising $13.87 \mathrm{~Gb}$ from the three libraries, with a Q30 percentage (sequencing error rate $0.1 \%$ ) above $82 \%$. The reads that only had 3 '-adaptor fragments were removed from the raw reads, resulting in $53.44 \mathrm{M}$ clean reads comprising $10.79 \mathrm{~Gb}$ with a Q30 above $94 \%$ (Table 1). The filtered reads were de novo assembled by Trinity (kmer length $=25$ ). The assembly results revealed that the transcriptome of E. breviscapus consists of 63,485 unigenes. The mean length of these unigenes was 882 bp and the N50 value was 1485 bp. The size distribution of the unigenes was shown in Fig. 1. The coding regions of each sequence were predicted by GetORF, which predicted 63,254 open reading frames (ORFs) from our assembled transcriptome, with 28,272 (44.7 \%) ORFs longer than $300 \mathrm{bp}$. The mean length of these ORFs was $554 \mathrm{bp}$ and the N50 value was $1167 \mathrm{bp}$. The size distribution of the unigenes is shown in Fig. 1. The high-quality reads produced in this study have been deposited in the NCBI SRA database (accession number: SRA245957).

\section{Functional annotation and classification}

Annotation of the assembled unigenes was based on searches of specific databases for sequence similarity. All of the unigenes were compared with sequences in the $\mathrm{Nr}$ database, the Swiss-Prot protein database, the KEGG database and the COG database, using BLASTX with a cutoff e-value of $10^{-5}$. A total of 38,540 unigenes (60.70\% of all unigenes) returned a significant BLAST result (Additional file 1: Table S1). Among them, 38,338 unigenes ( $60.38 \%$ of total unigenes) showed high similarity to sequences in the $\mathrm{Nr}$ database. The numbers of unigenes with significant similarity to sequences in the COG, KEGG and Swiss-Prot databases were 12,279 (19.34\%), 8483 (13.36 \%), and 25,994 (40.94\%), respectively (Table 2).

GO classification of the 28,571 annotated unigenes classified them into the functional categories: cellular components, molecular functions and biological processes. Among the various cellular components (ignoring unknown and other cellular component categories), cell, cell part and organelle were the most highly represented. Genes involved in other important cellular components, such as

Table 1 Quality of sequencing

\begin{tabular}{llllll}
\hline Samples & Raw reads & Clean reads & Clean bases & Q30 \% & GC \% \\
\hline T1 (control) & $24,614,951$ & $19,884,291$ & $4,016,227,333$ & 94.92 & 42.72 \\
T2 (self-) & $20,422,898$ & $15,554,953$ & $3,141,594,846$ & 94.56 & 43.39 \\
T3 (cross-) & $23,642,295$ & $18,001,474$ & $3,635,693,053$ & 94.69 & 43.30 \\
\hline
\end{tabular}

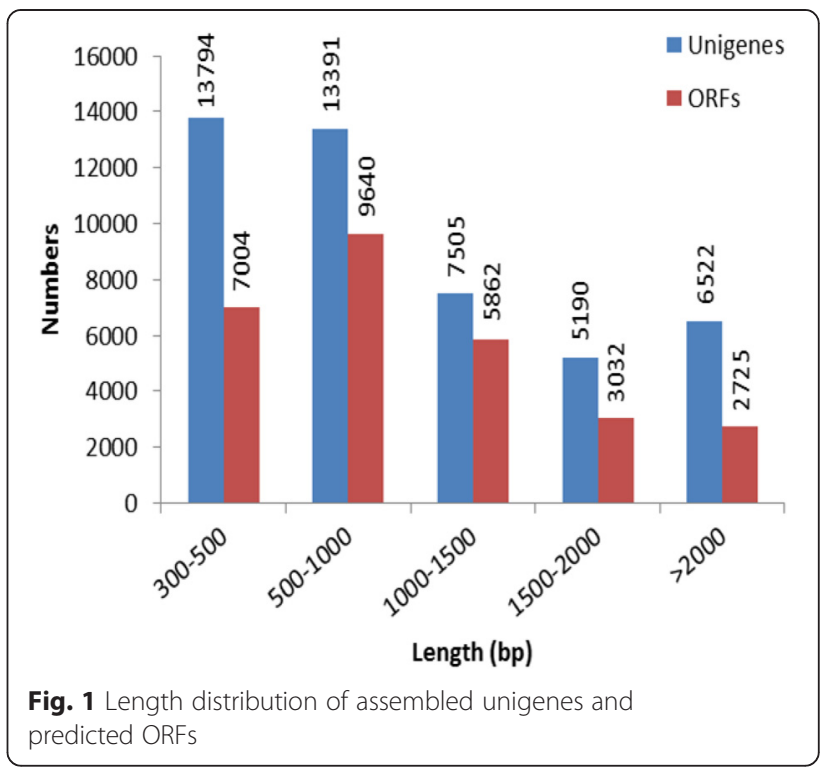

organelle parts, membrane, macromolecular complexes, extracellular regions, cell junction and membrane-enclosed lumen, were also identified through GO annotations. Similarly, binding and catalytic activities were most represented among various molecular functions; metabolic process and cellular process were most represented in the biological process categories (Fig. 2).

COG is a database built on phylogenetic relationships of protein sequences from 66 genomes, including bacteria, plants and animals. Individual proteins or paralogs from at least three lineages are categorized in each COG to represent an ancient conserved domain. Within the $E$. breviscapus unigenes dataset, 12,279 were categorized (E-value $\leq 1.0 \mathrm{E}-5$ ) into 25 functional COG clusters (Fig. 3). The five largest categories were: 1) General function prediction only; 2) Replication, recombination and repair; 3) Transcription; 4) Signal transduction mechanisms; and 5) Post-translational modification, protein turnover and chaperones.

\section{Gene expression differences in the three libraries}

Gene expression level was calculated using the RPKM method, which takes the influence of both the sequencing depth and gene length on read count into account. On the basis of the applied criteria [FDR $\leq 0.01$ and $\log _{2}$ (fold-change) $\geq 1$ ], 2072 genes were identified as significantly differentially expression genes (DEGs) between sample T1 (non-pollinated flowers) and T2 (self-pollinated flowers), in which 1426 were upregulated genes and 646 were downregulated in the E. breviscapus transcriptome. In addition, 2099 genes were identified as significant DEGs between samples T1 and T3 (crosspollinated flowers), and 145 genes were identified as 
Table 2 Summary of the annotation of unigenes of E. breviscapus

\begin{tabular}{lllll}
\hline & Sequences & Frequency & $\geq 300 \mathrm{nt}$ & $\geq 1000 \mathrm{nt}$ \\
\hline All assembled unigenes & 63,485 & $100.00 \%$ & 46,402 & 33,418 \\
Gene annotations against NR & 38,338 & $60.38 \%$ & 23,024 & 19,217 \\
Gene annotations against Swiss-Prot & 25,994 & $40.94 \%$ & 25,133 & 13,211 \\
GO annotation for NR protein hits & 28,571 & $45.00 \%$ & 11,479 & 7,451 \\
Gene annotations against COG & 12,279 & $19.34 \%$ & 13,575 & 7,906 \\
Gene annotations against KEGG & 8,483 & $60.70 \%$ & 33,547 & 18,639 \\
All annotated unigenes & 38,540 & & \\
\hline
\end{tabular}

significant DEGs between samples T2 and T3. For these DEGs, GO and KEGG analyses were performed.

Between samples T1 and T2, 1371 DEGs were associated with 50 subcategories, which were grouped into three major categories: biological processes, cellular components and molecular functions. In each of the three major categories of the GO classification, 'metabolic process', 'cell part' and 'catalytic activity' terms were dominant (Fig. 4). To further investigate the biochemical pathways of these DEGs, we mapped all DEGs to terms in the KEGG database. Of the 2072 DEGs, 220 genes had a KO ID and could be categorized into 78 pathways. Of those, two pathways were significantly enriched (corrected $\mathrm{P}$ value $\leq 0.05$ ): genes involved in plant-pathogen interaction and starch and source metabolism being the most significantly enriched (Fig. 5a). Between samples T1 and T3, the expression levels of 1452 genes were upregulated and 647 genes were downregulated; 1354 of these DEGs were associated with 52 sub-categories, and 230 were mapped to 81 pathways (Fig. 5b). Between samples $\mathrm{T} 2$ and T3, the expression levels of 92 genes were upregulated and 53 genes were downregulated; 73 of these DEGs were associated with 35 sub-categories, and 14 were mapped to 12 pathways (Fig. 5c).

\section{Comparison of transcriptional profiles of genes associated} with SI responses among the three libraries

Previous researches demonstrated that a number of genes involved in SI responses are differentially expressed, such as genes for cell-cell communication and signal transduction. To uncover genes involved in SI responses in E. breviscapus, the relative expression levels of SI-associated genes were analyzed in detail and the results demonstrated that most of their unigenes showed significant changes in expression levels in the three libraries. Those genes associated with SI

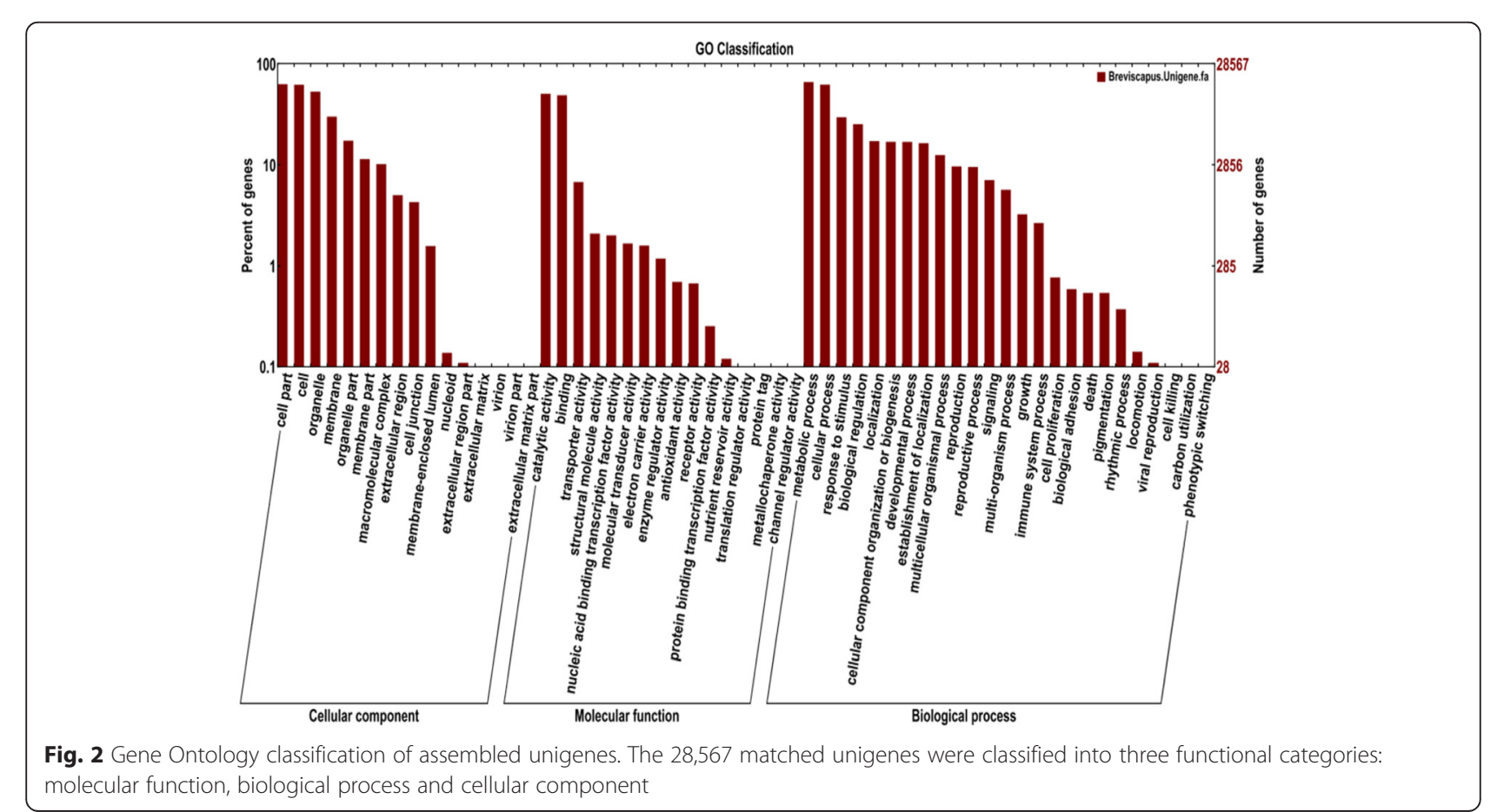




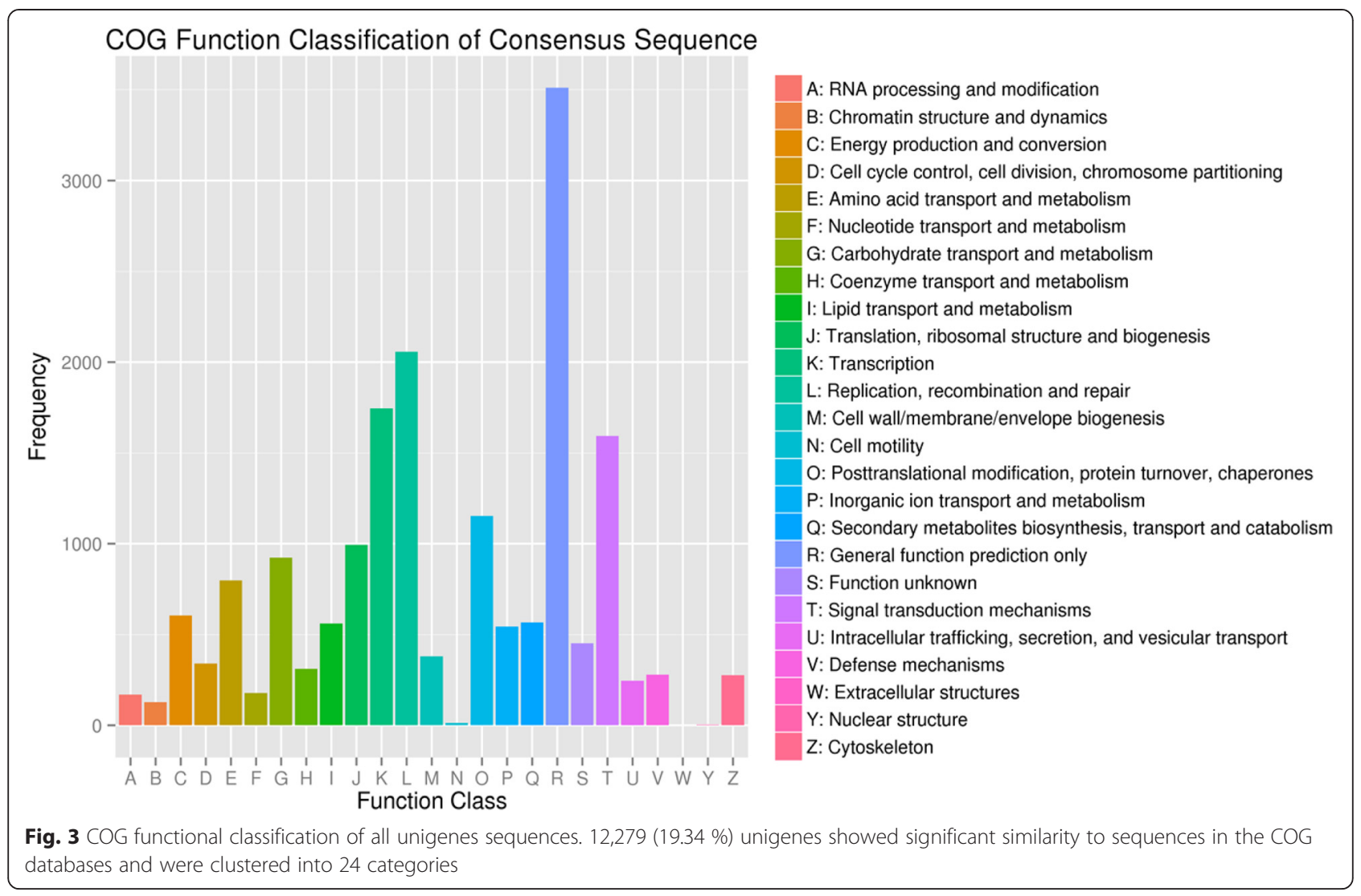

were clustered according to similarities in expression profiles between self- and cross-pollination. A heat map of gene expression of 230 putative genes involved in SI by pheatmap software is shown in Fig. 6 . Variance-stabilized data obtained using the DESeq package was used to generate the heat map by pheatmap software. The RNA-seq analysis of transcriptional data revealed that some genes (SRLK, MLPK and $K A P P)$ were highly expressed in self-pollinated capitulum but poorly expressed in cross-pollinated ones.

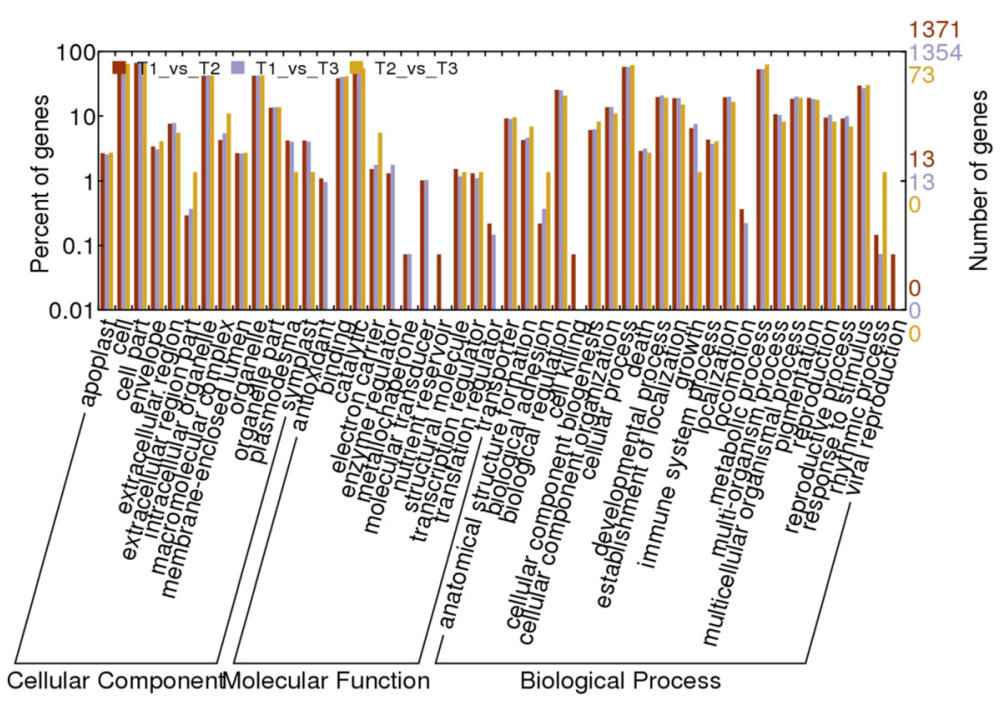

Fig. 4 Gene Ontology classification of differentially expressed genes between samples 


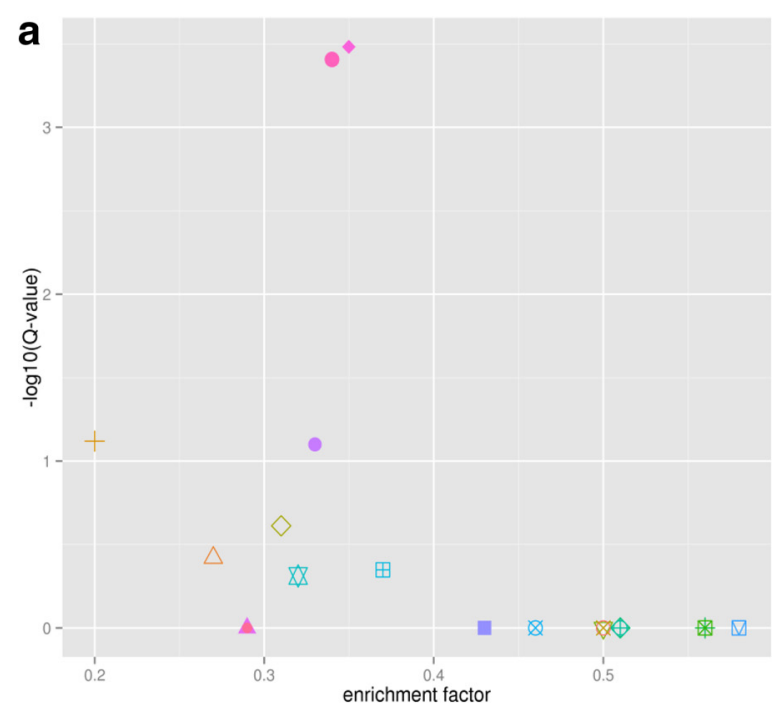

KEGG_pathway

Biosynthesis of unsaturated fatty acids

$\triangle$ Ether lipid metabolism

+ Flavonoid biosynthesis

$\times$ Fructose and mannose metabolism

Galactose metabolism

$\nabla$ Glycerolipid metabolism

$\triangle$ Glycerophospholipid metabolism

* Glycolysis / Gluconeogenesis

$\diamond$ Inositol phosphate metabolism

$\oplus \mathrm{N}-\mathrm{Glycan}$ biosynthesis

X Nitrogen metabolism

$\boxplus$ Pentose and glucuronate interconversions

$\otimes$ Pentose phosphate pathway

$\square$ Peroxisome

E Phenylalanine metabolism

- Phenylpropanoid biosynthesis

A Photosynthesis - antenna proteins

- Plant-pathogen interaction

- Starch and sucrose metabolism

- Zeatin biosynthesis

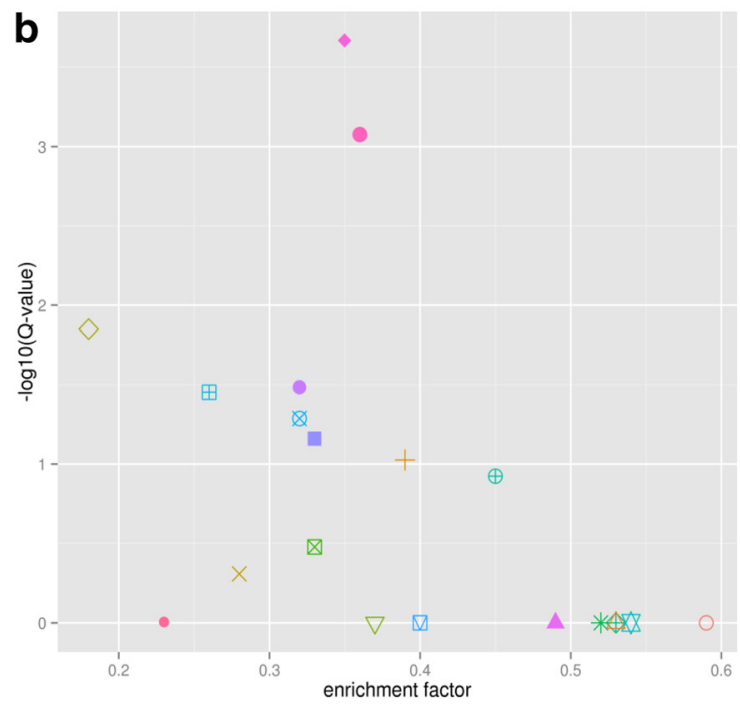

\section{KEGG_pathway}

Amino sugar and nucleotide sugar metabolism

$\triangle$ Biosynthesis of unsaturated fatty acids

+ Carbon fixation in photosynthetic organisms

$X$ Ether lipid metabolism

$\checkmark$ Flavonoid biosynthesis

$\nabla$ Fructose and mannose metabolism

$\triangle$ Galactose metabolism

* Glycerolipid metabolism

$\uparrow$ Glycerophospholipid metabolism

$\oplus$ Glycolysis / Gluconeogenesis

Inositol phosphate metabolism

$\boxplus$ Nitrogen metabolism

Q Pentose and glucuronate interconversions

$\square$ Pentose phosphate pathway

- Phenylalanine metabolism

- Phenylpropanoid biosynthesis

$\triangle$ Phosphatidylinositol signaling system

- Plant-pathogen interaction

- Starch and sucrose metabolism

- Zeatin biosynthesis

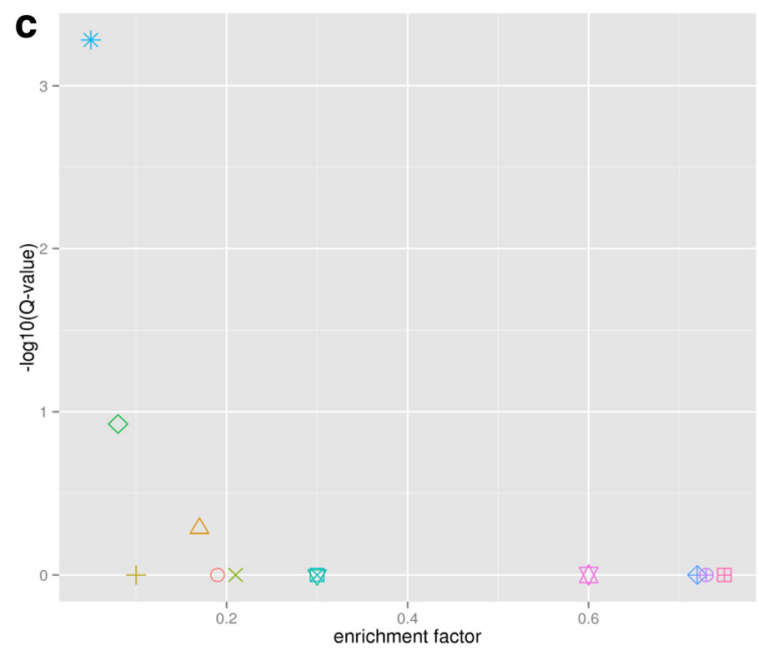

KEGG_pathway

$\triangle$ Biosynthesis of unsaturated fatty acids
Carbon fixation in photosynthetic organisms

+ Cyanoamino acid metabolism

$X$ Fatty acid metabolism

$\diamond$ Glyoxylate and dicarboxylate metabolism

$\nabla$ Oxidative phosphorylation

$\triangle$ Peroxisome

* Photosynthesis

$\diamond$ Plant hormone signal transduction

$\oplus$ Ribosome

XX RNA transport

$\boxplus$ Spliceosome

Fig. 5 a, $\mathbf{b}$ and $\mathbf{c}$ Scatterplot of differentially expressed genes enriched in KEGG pathways. The rich factor represents the ratio of the number of DEGs and the number of all the unigenes in the pathway; the $\mathrm{Q}$ value represents the corrected $P$-value 


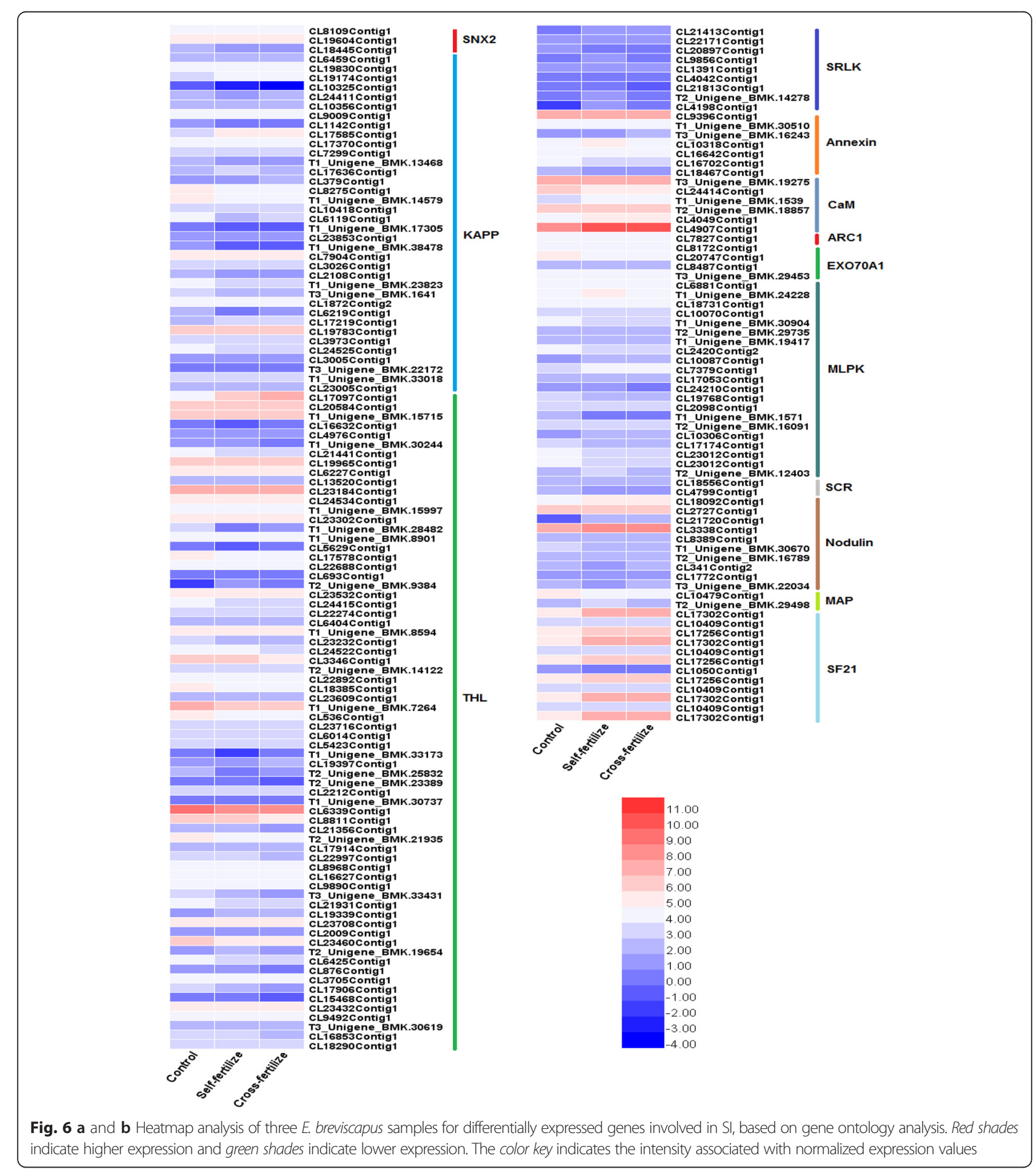

In contrast, $T H L$ and $C a M$ were downregulated after self-pollination but upregulated after cross-pollination. However, Exo70A1 showed no difference among three cDNA libraries. These genes will be further examined to investigate their biological functions. The RPKM value of partial unigenes involved in SI responses were listed in Additional file 2: Table S2.
Identification and expression analysis of candidate genes involved in SI responses

A putative SRK-like gene, EbSRLK 1 (CL21813Contig1), was cloned. Phylogenetic analysis was performed by alignment using entire predicted protein sequences from E. breviscapus and other species by mega software. The neighbor-joining phylogenetic tree demonstrated that 
CL21813contig1 (EbSRLK1) is most closely related to SRK from S. squalidus L., but is distant from the SRK of crucifer species (GenBank accession numbers: CAG28412, CAG28414 and CAG28413) (Fig. 7). Calcium ions are able to transmit diverse signals that exert primary actions on cells. Calmodulin ( CaM), as the multifunctional calcium receptor, is associated with various physiological and developmental processes in plants. The RNAseq results showed that the expression levels of CL21813contig1 (EbSRLK1) and T3_Unigene_BMK.9975 (EbSRLK3) were upregulated after self-pollination. The highest expression levels were observed at 24 and $10 \mathrm{~h}$ after self-pollination for EbSRLK1 and EbSRLK3, respectively. However, their expression levels were lower in cross-pollinated capitulum than in self-pollinated capitulum. The highest expression levels for EbSRLK1 and EbSRLK3 in cross-pollinated capitulum were observed at 24 and $48 \mathrm{~h}$ after pollination, respectively. CL4907Contig1 $(E b C a M)$ was downregulated after self-pollination and upregulated after cross-pollination. The expression levels of the three genes were significantly different between self- and cross-pollination (repeated-measures ANOVA: EbSRLK1 time effect, $F_{1,16}=96.822$, $P<0.001$. time * treatment, $F_{7,16}=100.492, P<0.001$; treatment, $F_{7,16}=34.321, P<0.001 ; E b S R L K 3$ time effect, $F_{1,16}=16.929, P=0.001$. time * treatment, $F_{7,16}$ $=212.676, P<0.001 ;$ treatment, $F_{7,16}=112.076, P<$ 0.001; $E b C a M$ time effect, $F_{1,16}=14.695, P=0.001$. time "treatment, $F_{7,16}=18.906, P<0.001$; treatment, $\left.F_{7,16}=20.065, \quad P<0.001\right)$. Furthermore, the expressions of the three genes were significantly different at $6,10,24$ and $48 \mathrm{~h}$ after self- and cross-pollination (EbSRLK1 independent $T$-test: $t \geq 5.650, \quad P \leq 0.005$; EbSRLK3 $t \geq 9.740, \quad P \leq 0.001 ;$ EbCaM $t \geq 6.208$, $P \leq 0.003)$. To confirm the results of RNAseq, three candidate SI-associated genes (two EbSRLKs and one $\mathrm{CaM}$ ) were chosen for further expression analysis. Using gene-specific primer pairs, the expression levels of the candidate genes were analyzed at different time points after self- or cross-pollination by using qRTPCR (Fig. 8). The expression patterns of these three genes in the qRT-PCR analysis showed the similar trend as the RNAseq analysis (Fig. 8).

\section{Discussion}

To identify genes involved in the SI, we sequenced the E. breviscapus transcriptome and de novo assembled reads from next-generation RNA-seq data. We identified 63,485 unigenes in the three libraries (Sample T1 for non-pollinated flowers, Sample T2 for selfpollinated flowers, and Sample T3 for cross-pollinated flowers). In the absence of E. breviscapus genomic information, the availability of the transcriptome data will provide a valuable resource to investigate the mechanisms of SI responses.

\section{Genes involved in SI responses in E. breviscapus}

The $S$-locus encodes two proteins: SRK and SCR. SRK encodes an $S$ receptor kinase [42, 43]. E. breviscapus exhibits an SSI system of SI as other Asteraceae species. $S$ alleles have high amino acid sequence divergence within species, and E. breviscapus is no exception: the pistil $S R L K$ genes from the transcriptome showed sequence divergence. Phylogenetic analysis of these unigenes with other SRKS from different species demonstrated that the characteristics of some SRKS (such as T1_unigene_BMK.9975 and CL21413Contig1) in E. breviscapus are not consistent with other SRK genes (not shown). The RNA-seq analysis of transcriptional data revealed the same expression pattern (Fig. 6b). Previous studies of $S$. squalidus have shown that orthologues of the Brassica $S$-gene, $S R K$, are not expressed exclusively in the stigma, or linked to the $S$-locus [21]. In the three samples, differentially expressed, putative EbSRLK genes were identified. One putative EbSRLK1 gene (CL21813contig1) was cloned. Quantitative PCR analysis revealed that EbSRLK1 was highly expressed in selfpollinated capitulum and poorly expressed in crosspollinated ones (Fig. 8). However, their expression patterns suggested that they are unlikely to be directly involved in SI and which may be similar to the SRK-like genes in S. squalidus. Further studies are needed to dissect the functions of these SRK-like genes in $E$. breviscapus.

In contrast to the female factor SRKs, the male $S$ determinant SCR acts as a ligand [44]. The HV region in the $S$-domain of SRKs acts as the SCRbinding site, which involves a two-stage recognition process [45]. In the three libraries of E. breviscapus, only two unigenes were annotated as SCR, CL18556Contig1 and CL4799Contig1 (Fig. 6b). Functional analysis of the role of SCRs in E. breviscapus during pollen-pistil interaction will be performed in a future study.

\section{Signaling pathway for the SI reaction}

The E. breviscapus dataset revealed many genes that play primary roles in the recognition between the stigma and pollen grains. Among the identified genes, we focused on those genes encoding MAP, SF21, Nodulin3, ARC1, MLPK, EXo70A1, CaM and THL1/THL2.

$A R C 1$ and $M L P K$ are candidate downstream effectors of $S R K$. therefore, $A R C 1$ might be a positive effector in the SI response in Brassica. Antisense suppression of $A R C 1$ led to partial breakdown of the SI response [46]. In the three samples in this study, only one $A R C 1$ transcript was found (Fig. 6b). The absence of other ACR1 


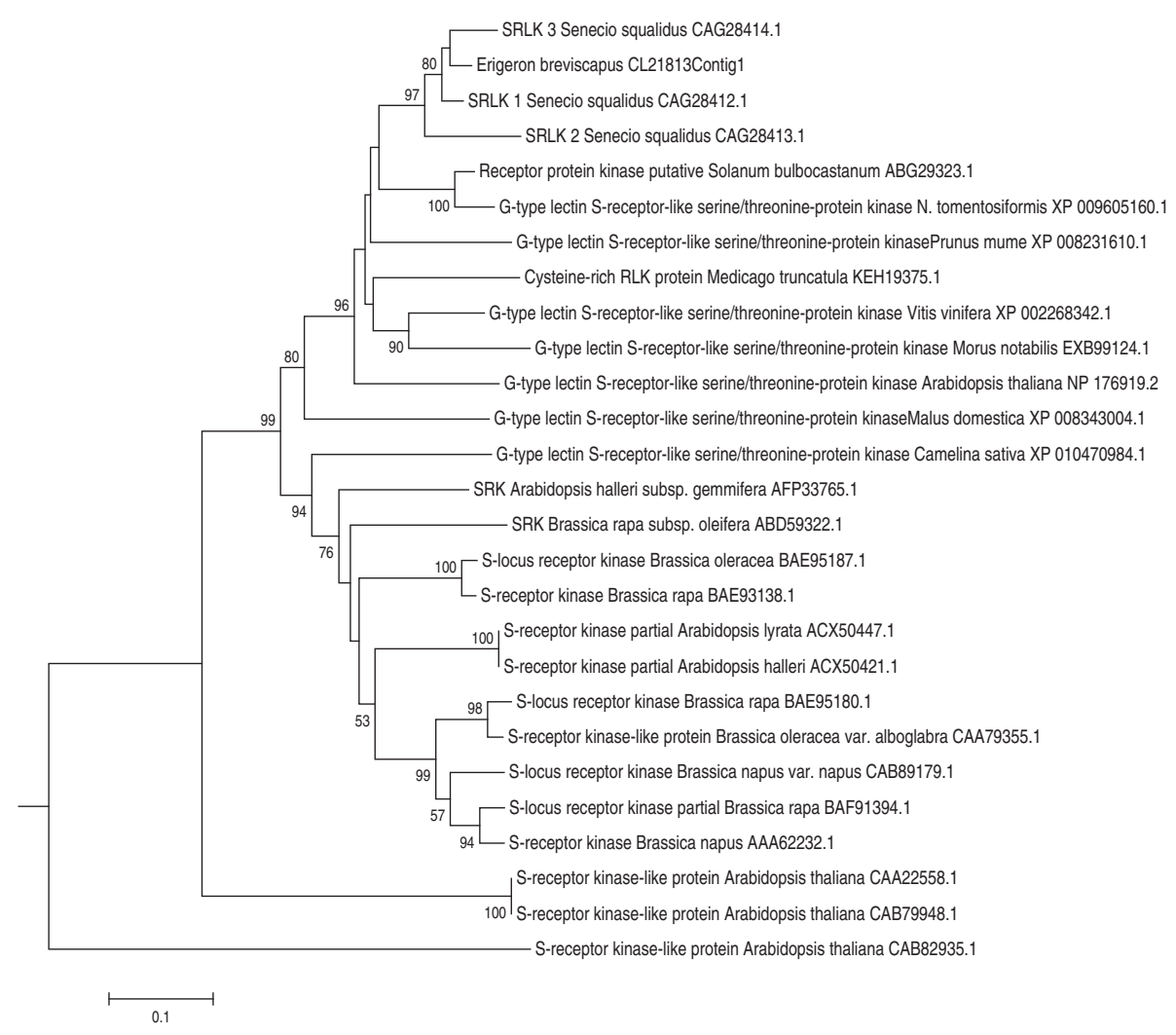

Fig. 7 Phylogenetic analysis of CL21813contig1 from E. breviscapus with other SRKs from different species. The neighbor-joining tree based on p-distance and pairwise deletions of gaps indicates the phylogenetic relationships among the putative SRK proteins in different species. The numbers at the branching points indicate the bootstrap support values (200 replicates). The putative E. breviscapus $S$ locus receptor protein kinase is marked with a triangular symbol

genes suggest that the assemblies were incomplete and/ or that expression of the ACR1 genes is too low to acquire enough reads to be assembled. $M L P K$ can interact with the kinase domain of SRK [47]. MPLK is thought to function in SRK-mediated signaling. In the present study, we found that the $M L P K$ gene was upregulated in the self-pollinated and downregulated in cross-pollinated sample (Fig. 6b).

As a factor interacting with $A R C 1, E x o 70 A 1$ has been isolated in B. napus [48]. Overexpression of Exo70A1 in self-incompatible $B$. napus partially breaks down SI, whereas suppression of Exo70A1 (by RNAi or T-DNA insertion) in self-compatible $B$. napus and $A$. thaliana inhibited pollen adhesion, hydration and germination [48]. Six unigenes encoding Exo70A1 were identified in our study, although no differences in their expressions were observed among three cDNA libraries (Fig. 6b). Further studies are required to clarify the function of Exo70A1 genes in E. breviscapus.

THL1 and THL2 were identified as SRK-binding partners from a yeast two-hybrid screen [49] and which act as negative regulators in SRK signaling $[50,51]$. Studies on the Brassicaceae showed that THL plays a key role in
SI response. In the three cDNA libraries of E. breviscapus, one striking finding was the identification of $88 \mathrm{pu}-$ tative THL proteins (Fig. 6a). Functional analysis will be necessary to shed light on the role of the THL1/THL2 during signal transduction in E. breviscapus.

Calmodulin is an important second messenger in many signal transduction pathways and an important calcium-banding protein. CL4907Contig1 (EbCaM) was downregulated after self-pollination and upregulated after cross-pollination (Fig. 8). Quantitative PCR analysis showed that the expression pattern of the $E b C a M$ gene was the same as in the RNA-seq analysis (Fig. 6a and b).

Some new SI candidate genes, MAP, SF21, Nod, were identified based on the fact that they specifically express in pistils of $S$. squalidus. The nucleotide sequence of $M A P$ exhibits relatively high $S$-genotypic polymorphism [22]. In the three samples in E. breviscapus, few MAP transcript was found. The reason for the deletion of $M A P$ gene may be similar to $A R C 1$ gene, i.e., the assemblies were incomplete and/or that expression of the $M A P$ genes is too low to acquire enough reads to be assembled (Fig. 6b). Phylogenetic analysis of SF21 nucleotide sequence alignments indicate that this gene family 


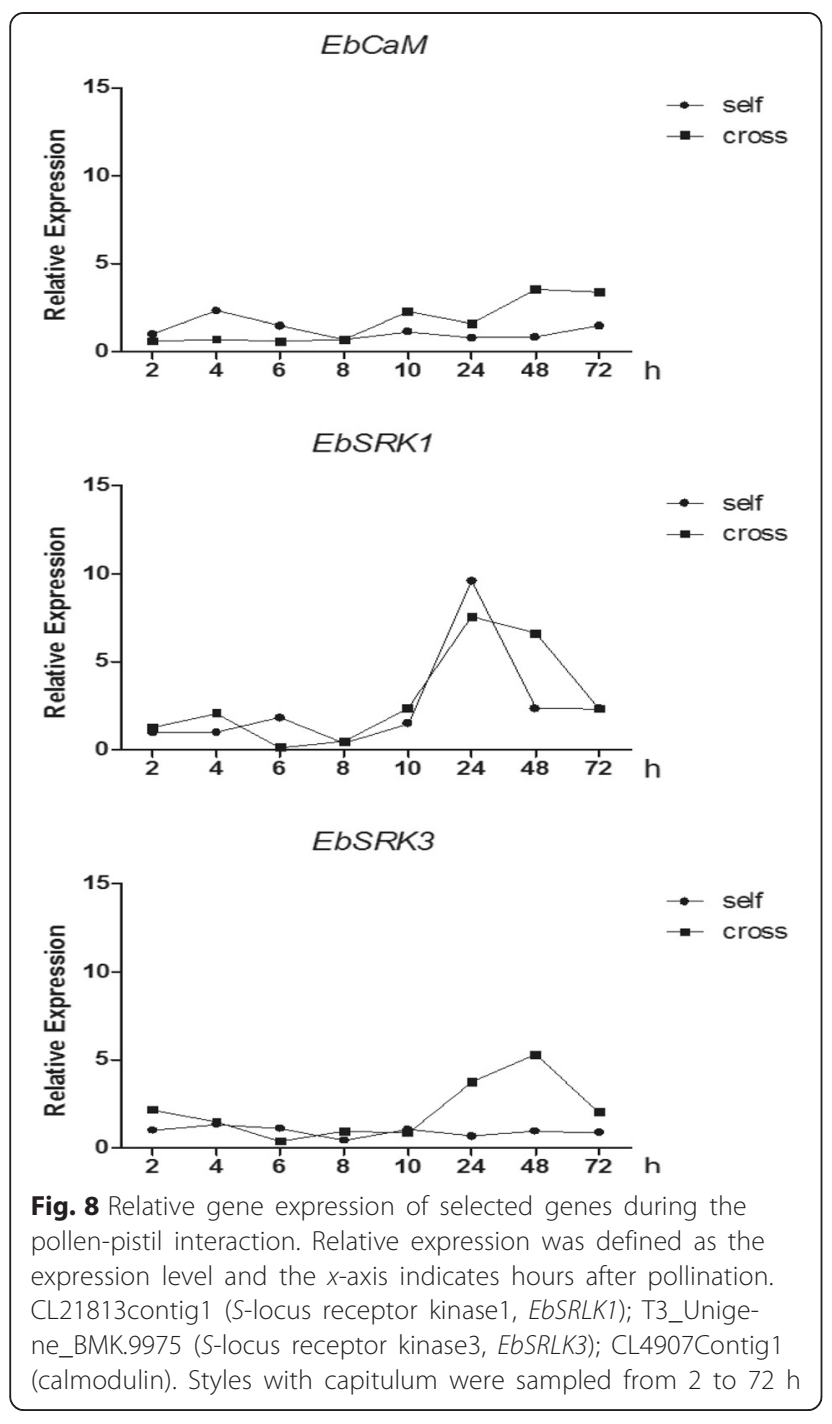

is conserved and may play an important role in reproductive processes in flowering plants. In E. breviscapus transcriptome dataset, we found that the SF21 genes were upregulated in the self-pollinated and crosspollinated sample (Fig. 6b). Nod gene family has been investigated in Arabidopsis and rice, which have a crucial function in pollen development [52, 53]. Despite the large number of pistil-specific nodulin/mtn3 genes have been investigated, the function of these genes in pistils has not been studied. Ten unigenes encoding Nod were identified in our study, they were also upregulated in the self-pollinated and cross-pollinated sample (Fig. 6b). SF21 and Nod might well be involved in SI, however, further studies are needed.

Moreover, there were many other genes that might be involved in $\mathrm{SI}$ in the three libraries of E. breviscapus, such as SNX2, KAPP and Annexin (Fig. 6a and b). Further studies are required to fully understand the role and mechanism of these candidate genes in SI.

\section{Conclusions}

We performed the first large-scale investigation of gene expression in the capitulum of E. breviscapus, an Asteraceae SSI species, using high-throughput RNA-seq analysis. After assembly, 63,485 gene models were obtained (average gene size $882 \mathrm{bp}$; N50 = $1485 \mathrm{bp}$ ), among which 38,540 unigenes (60.70 \% of total gene models) were annotated by comparing them with four public databases (Nr, Swiss-Prot, KEGG and COG). 38,338 unigenes $(60.38 \%)$ showed high similarity with sequences in the $\mathrm{Nr}$ database. DEGs were identified among the three cDNA libraries (non-, self- and cross-pollinated capitulum of E. breviscapus). Approximately 230 genes showed differential expression that might be associated with SI. Quantitative PCR confirmed the expression patterns of selected genes examined by RNA-seq. Most of the genes identified by the RNA-seq analysis were not previously reported or studied in E. breviscapus. Although function information is missing, we hypothesized that MLPK, ARC1, CaM, Exo70A1, MAP, SF21 and Nod might be crucial for the SI responses in E. breviscapus. However, EbSRLK1 and EbSRLK3 genes were found not closely related to SI in E. breviscapus and they are more like the SRK-like genes. The results will lead to a better understanding of the SSI system in the Asteraceae. The functions of these genes will provide clues to the mechanisms that underlie SI in E. breviscapus SSI systems.

\section{Methods}

\section{Plant materials and RNA isolation}

Wild-type of E. breviscapus were used in this study. Flower tissues of E. breviscapus were collected from an experimental plot in the planting base (Luxi County, Yunnan, China). We have got permission from the sample provider. Experiments of forced self-pollination and cross-pollination were performed at 3rd April 2014. In short, plants were prepared for experiments by bagging branches bearing developing flower buds. All pollinations (selfs and crosses) were repeated for at least three times and at least ten bagged flowering heads (capitula) were collected. Controlled self- and cross-pollinations were performed as previously described in Hiscock [54] and Brennan et al. [23, 25]. In cross treatment, the anthers were removed by a sable paint brush. Crosspollinations were carried out by touching stigmas with a capitulum containing flowers with pollen fully presented. Following pollination, capitula were secured within a small, porous, tissue pollination bag $(5 \mathrm{~cm} \times 5 \mathrm{~cm})$ using a pin. On the other hand, in forced-selfing, selfing solution $(1 \% \mathrm{NaCl}$ in $10.1 \%$ Tween 20$)$ was applied to stigmas of bagged capitula with a dry paint brush. Stigmas were allowed to dry for some times, then applying selfpollen from another capitulum from the same individual and re-bagging selfed capitula. Self-pollination was 
enhanced by occasional agitation of bagged capitula. Three samples were harvested at $24 \mathrm{~h}$ after non(Sample T1), self- (Sample T2) and cross-pollination (Sample T3).

For Illumina sequencing, total RNA was extracted using an EASY spin microRNA Rapid extraction Kit (Aidlab, Beijing, China). Both the quantity and quality of the total RNA were verified using a NanoDrop ND1000 (Thermo Scientific), gel electrophoresis and an Agilent 2100 Bioanalyzer (Agilent). Thirty micrograms of RNA was pooled equally from 10 capitulum for cDNA library preparation.

\section{RNA-seq library construction and sequencing}

The cDNA library was constructed using a cDNA Sample Preparation Kit (Cat \# RS-930-1001, Illumina Inc., San Diego, CA), following the manufacturer's instructions. The total RNA was collected and pooled from each treatment, and mRNA was enriched and purified using poly- $\mathrm{T}$ oligo nucleotide-attached magnetic beads. The mRNA was cleaved using an RNA Fragmentation Kit (Ambion, Austin, TX, USA) and then used as templates for first-strand cDNA synthesis using reverse transcriptase (Invitrogen, Carlsbad, CA, USA) and random hexamer primers. Second-strand cDNA was synthesized using DNA polymerase I (New England BioLabs, Ipswich, MA). The cleaved fragments were purified using a MinElute PCR Purification Kit (Qiagen, Dusseldorf, Germany) and ligated to Solexa adaptors. The desired fragments (ca. 150-200 bp in size) were excised from $1.8 \%$ agarose gels and purified using a Gel Extraction Kit (Qiagen). Finally, PCR amplicons were purified and the sequencing library was successfully constructed. The Illumina HiSeq 2000 sequencing platform was used for sequencing. The transcriptome datasets were analyzed by Illumina software.

\section{De novo transcriptome assembly}

After sequencing, raw reads that contained adapters, reads containing more than $5 \%$ of unknown sequences ('N'), and low-quality bases that were identified based on CycleQ30 (corresponding to a $0.1 \%$ sequencing error rate) were removed before assembly. De novo assembly of the clean reads was performed using the Trinity program [55]. First, reads with a certain length of overlap were combined to form contigs. They were then mapped back to contigs with paired-end reads to detect contigs from the same transcript, as well as the distances between contigs. Finally, Trinity connected contigs and obtained sequences that could not be extended on either end. Such sequences were defined as 'unigenes'. After that, unigenes from the three libraries were further assembled to obtain non-redundant unigenes using the TGICL software and non-redundant unigenes were used for further analysis [56]. Potential coding regions within the unigenes were analyzed using the GetORF function in the EMBOSS software package. Length distribution was analyzed using common perl scripts. The N50 length, mean length and unigene number with different length intervals were all calculated.

\section{Functional annotation and KEGG pathway analysis}

The functions of the unigenes were annotated using BLASTX [57] searches with an E-value threshold of $10^{-5}$ against protein databases, including the NCBI nonredundant $(\mathrm{Nr})$ database (http://www.ncbi.nlm.nih.gov) [58], the Swiss-Prot protein database (http://www.expasy.ch/sprot) [59], the Kyoto Encyclopedia of Genes and Genomes (KEGG) database (http://www.genome.jp/ kegg) [60], and the Clusters of Orthologous Groups of proteins (COG) database (http://www.ncbi.nlm.nih.gov/ COG) [61]. The proteins with the highest sequence similarity were retrieved for analysis. KEGG produced annotations of metabolic pathways, while COG matched each annotated sequences to a conserved domain.

\section{Abundance estimation and differential expression analysis}

To investigate the expression level of each unigene in different samples, transcript abundance estimates were obtained by running RNA-seq by ExpectationMaximization analysis separately for each sample, which uses an iterative process to fractionally assign reads to each transcript based on the probabilities of the reads being derived from each transcript [62]. The alignment produced digital expression levels for each unigene, which were normalized using perl scripts in the Trinity package to obtain reads per kilo base per million (RPKM) values [63].

To study the expression patterns of the transcripts across samples, it is often useful to restrict analysis to those transcripts that are significantly differentially expressed in at least one pairwise sample comparison. Differential expression analysis of the three samples was performed using tools from the Bioconductor project, including edgeR [64] and DESeq [65], to identify differentially expressed transcripts. Given a set of differentially

Table 3 Primers used for gene validation and expression analyses

\begin{tabular}{lll}
\hline Primers & Sequences & Size (bp) \\
\hline EbSRLK1F & GGGCAGAATCGGACCCTTAC & 95 \\
EbSRLK1R & TGAGACCCTTCTTCGTTGG & 95 \\
EbSRLK3F & CTAGGCTGTTGCATTCGTGG & 293 \\
EbSRLK3R & GGTGATTGCTTCAGTCTCATTTGG & 293 \\
EbCaM-F & AGGCTGAACTCCAAGACAT & 118 \\
EbCaM-R & TCCTCCTCAGAGTCCGTAT & 118 \\
\hline
\end{tabular}


expressed transcripts, we extracted those transcripts with an FDR (false discovery rate) $\leq 0.01$ and $\log _{2}$ foldchange $\left(\log _{2} \mathrm{FC}\right) \geq 1$.

\section{Gene validation and expression analyses}

To investigate different gene (EbSRLK1, EbSRLK3 and $E b C a M)$ expressions in E. breviscapus, eight flower stages $(2,4,6,8,10,24,48$ and $72 \mathrm{~h}$ after pollination) were collected and examined. qRT-PCR was performed with gene-specific primers. All primers used in this study are listed in Table 3. Total RNA was extracted from self-pollinated, cross-pollinated, or non-pollinated (control) capitulum in E. breviscapus using the TRIzol Reagent (Takara, Beijing, China). RNA was further purified using an RNA purification kit (Takara). In this experiment, GAPDH was chosen as the housekeeping gene. Each reaction was performed in triplicate (each biological replicate contained tissues from at least ten capitulum) and the experiment was repeated three times (technical replicates) for each sample. The standard curve method was used to determine the mRNA relative expression levels, which were normalized to the reference gene. Real-time PCR was performed in a Roche detection system (Roche, Switzerland). The thermal cycler parameters were used as follow: $30 \mathrm{~s}$ at $94{ }^{\circ} \mathrm{C}$; then $45 \mathrm{cy}$ cles of $20 \mathrm{~s}$ at $94{ }^{\circ} \mathrm{C}, 20 \mathrm{~s}$ at $55^{\circ} \mathrm{C}$, and $30 \mathrm{~s}$ at $72{ }^{\circ} \mathrm{C}$. The melting curve analysis was carried out from $60{ }^{\circ} \mathrm{C}$ to $95{ }^{\circ} \mathrm{C}$ to observe the specificity of the PCR products. Data were analyzed using repeated-measures ANOVA, using time $(2,4,6,8,10,24,48$ and $72 \mathrm{~h})$ as the withinsubject effect and different mating patterns (self- and cross-pollination) as the between-subject effect, respectively. Independent $\mathrm{t}$-tests were performed to explore the detailed effects of treatment. All calculations were performed using SPSS Statistics 17.0 (www.spss-china.com).

\section{Availability of supporting data}

The supporting data such as the high-quality reads produced in this study have been deposited in the NCBI SRA database, an open access repository (accession number: SRA245957).

\section{Additional files}

Additional file 1: Table S1. Functional annotation of E.breviscapus transcriptome. (XLSX $4281 \mathrm{~kb})$

Additional file 2: Table S2. The RPKM value of partial unigenes. (XLSX $520 \mathrm{~kb})$

\section{Abbreviations}

SI: Self-incompatibility; GSI: Gametophytic self-incompatibility;

SSI: sporophytic self-incompatibility; ORF: Open reading frames; RPKM: Reads Per Kilobase of exon model per Million mapped reads; FDR: False discovery rate; DEGs: Differentially expression genes; BLAST: Basic Local Aligment Search Tool; Go: Gene Ontology; KEGG: The KEGG resource for deciphering the genome; COG: Clusters of orthologous Groups of proteins; Nr: ncbi nr database; qRT-PCR: Quantitative real time polymerase chain reaction; E. breviscapus: Erigeron breviscapus; SRLK: S-locus receptor-like kinase; SCR: Slocus cysteine-rich; MLPK: M-locus protein kinase; ARC1: Arm repeat containing 1; CaM: Calmodulin; MAP: Membrane associated protein; SF21: Sunflower-21; Nod: Nodulin/mtn3; THL1: Thioredoxin-like protein 1; THL2: Thioredoxin-like protein 2; KAPP: Kinase associate protein phosphatase; SNX2: Annexin S2; Swiss-Prot: A high quality annotated and non-redundant protein sequence database; edger: a Bioconductor package for differential expression analysis of digital gene expression data Bioinformatics; DESeq: Different gene expression analysis based on the negative binomial distribution; FDR: False discovery rate.

\section{Competing interests}

The authors declare that they have no competing interests.

\section{Authors' contributions}

WZ and XW carried out differential gene expression analysis, GRT-PCR and drafted the manuscript. NHJ,HLM and CHM participated in RNA-seq analysis. $\mathrm{GHZ}$ and SCY initiated the project and supervised the work throughout. All authors read and approved the final manuscript.

\section{Authors' information}

Not applicable.

Availability of data and materials Not applicable.

\section{Acknowledgements}

We would like to thank the Institute of Herba Erigerontis of Honghe Prefecture/Honghe Qianshan Bioengineering Co., Ltd for providing the Erigeron breviscapus sample, and Zhiwen Gong for advice on qRT-PCR analysis.

\section{Funding}

This work was supported by the National Natural Science Foundation of China (No.81160499 and No. 81503184).

\section{Author details}

${ }^{1}$ Yunnan Research Center on Good Agricultural Practice for Dominant Chinese Medicinal Materials, Yunnan Agricultural University, Kunming 650201, Yunnan, People's Republic of China. ${ }^{2}$ The Life Science and Technology College, Honghe University, Mengzi 661100, Yunnan, People's Republic of China. ${ }^{3}$ Key Laboratory of Tropical Agro-environment, Ministry of Agriculture, South China Agricultural University, Guangzhou 510642, People's Republic of China.

Received: 9 April 2015 Accepted: 23 September 2015

Published online: 13 October 2015

\section{References}

1. de Nettancourt D. Incompatibility and incongruity in wild and cultivated plants. 2nd ed. Berlin: Springer Verlag; 2001.

2. Dickinson HG, Elleman CJ, Doughty J. Pollen coatings chimaeric genetics and new functions. Sex Plant Reprod. 2000;12:302-9.

3. Takayama S, Isogai A. Self-incompatibility in plants. Annu Rev Plant Biol. 2005;56:467-89

4. Takayama S, Shimosato H, Shiba H, Funato M, Che FS, Watanabe M, et al. Direct ligand-receptor complex interaction controls Brassica selfincompatibility. Nature. 2001;413:534-8.

5. Franklin-Tong VE, Holdaway-Clarke TL, Straatman KR, Kunkel JG, Hepler PK. Involvement of extracellular calcium influx in the self-incompatibility response of Papaver rhoeas. Plant J. 2002;29:333-45.

6. Loerger TR, Clark AG, Kao TH. Polymorphism at the self-incompatibility locus in Solanaceae predates speciation. Proc Natl Acad Sci U S A. 1990:87:9732-5.

7. Charlesworth D. Multi-allelic self-incompatibility polymorphisms in plants. Bioessays. 1995:17:31-8.

8. Jung HJ, Park Jl, Ahmed NU, Chung MY, Kim HR. Characterization of self-incompatibility genes in the intergeneric hybrid $\times$ Brassic oraphanus. Plant Syst Evol. 2014;300:1903-11. 
9. Jung HJ, Ahmed NU, Park Jl, Thamilarasan SK, Kim HR, Cho YG, et al. Analysis of S-locus and expression of S-alleles of self-compatible rapidcycling Brassica oleracea 'TO1000DH3'. Mol Biol Rep. 2014;41:6441-8.

10. Nasrallah JB, Chen $\mathrm{CH}$, Kao TH, Goldberg ML, Nasrallah ME. Amino-acid sequence of glycoproteinsencoded by three alleles of the $S$ locus of Brassica oleracea. Nature. 1987;326:617-9.

11. Suzuki G, Kai N, Hirose T, Fukui K, Nishio T, Takayama S, et al. Genomic organization of the $S$ locus: identification and characterization of genes in SLG/SRK region of S(9) haplotype of Brassica campestris (syn. rapa). Genetics. 1999;153:391-400.

12. Schopfer CR, Nasrallah ME, Nasrallah JB. The male determinant of selfincompatibility in Brassica. Science. 1999;286:1697-700.

13. Gu T, Mazzurco M, Sulaman W, Matias DD, Goring DR. Binding of an arm repeat protein to the kinase domain of the S-locus receptor kinase. Proc Natl Acad Sci U S A. 1998:95:382-7.

14. Muzzurco M, Sulaman W, Elina H, Cock JM, Goring DR. Further analysis of the interactions between the Brassica $S$ receptor kinase and three interacting proteins (ARC1, THL1 and THL2) in the yeast two-hybrid system. Plant Mol Biol. 2001;45:365-76.

15. Murase K, Shiba H, Iwano M, Che F-S, Watanabe M, Isogai A, et al. A membrane anchored protein kinase involved in Brassica self-incompatibility signaling. Science. 2004;303:1516-9.

16. Caruso M, Merelo P, Distefano G, La Malfa S, Piero ARL, Tadeo FR, et al. Comparative transcriptome analysis of stylar canal cells identified novel candidate genes implicated in the self-incompatibility response of Citrus clementina. BMC Plant Biol. 2012;12:20.

17. Hughes MB, Babcock EB. Self-incompatibility in Crepis oetida L. subsp. rhoeadifolia Bieb. Schinz et Keller. Genetics. 1950;35:570-88.

18. Gerstel DU. Self-incompatibility studies in Guayule. Genetics. 1950;35:482-506.

19. Elleman CJ, Franklin-Tong VE, Dickinson HG. Pollination in species with dry stigmas: the nature of the early stigmatic response and the pathway taken by pollen tubes. New Phytol. 1992;121:413-24.

20. Hiscock SJ, Hoedemaekers K, Friedman WE, Dickinson HG. The stigma surface and pollen-stigma interactions in Senecio squalidus $L$ (Asteraceae) following cross (compatible) and self (incompatible) pollinations. Int J Plant Sci. 2002;163:1-16.

21. Hiscock SJ, Mclnnis SM, Tabah DA, Henderson CA, Brennan AC. Sporophytic self-incompatibility in Senecio squalidus L. (Asteraceae)-the search for S. J Exp Bot. 2003;54:169-74.

22. Allen AM, Lexer C, Hiscock SJ. Comparative analysis of pistil transcriptomes reveals conserved and novel genes expressed in dry, wet, and semidry stigmas. Plant Physiol. 2010;154:1347-60.

23. Brennan AC, Harris SA, Tabah DA, Hiscock SJ. The population genetics of sporophytic self-incompatibility in Senecio squalidus L. (Asteraceae) I: $S$ allele diversity in a natural population. Heredity. 2002;89:430-8.

24. Brennan AC, Harris SA, Hiscock SJ. The population genetics of sporophytic self-incompatibility in Senecio squalidus L. (Asteraceae) avoidance of mating constraints imposed by low S-allele number. Philos Trans R Soc Lond B Biol Sci. 2003;358:1047-50.

25. Brennan AC, Harris SA, Hiscock SJ. The population genetics of sporophytic self-incompatibility in Senecio squalidus L. (Asteraceae): the number, frequency and dominance interactions of $S$ alleles across its British range. Evolution. 2006;60:213-24.

26. Allen AM, Lexer C, Hiscock SJ. Characterisation of sunflower-21 (SF21) genes expressed in pollen and pistil of Senecio squalidus (Asteraceae) and their relationship with other members of the SF21 gene family. Sex Plant Reprod. 2010;23:173-86.

27. Allen AM, Thorogood CJ, Hegarty MJ, Lexer C, Hiscock SJ. Pollen-pistil interactions and self-incompatibility in the Asteraceae: new insights from studies of Senecio squalidus (Oxford ragwort). Ann Bot. 2011;108:687-98.

28. Li L, Dang CL. Flora syndrome and breeding system of Erigeron breviscapus. Acta Ecol Sin. 2007;27:571-8.

29. Song KX, Wang YH, Yi TS, Yang ZY. Karyological studies of Erigeron breviscapus and related species. Caryologia. 2010;63:176-83.

30. Chu $\mathrm{Q}, \mathrm{Wu} T, \mathrm{Fu} \mathrm{L}, \mathrm{Ye} J$. Simultaneous determination of active ingredients in Erigeron breviscapus (Vant.)Hand-Mazz. by capillary electrophoresis with electrochemical detection. J Pharm Biomed Anal. 2005;37:535-41.

31. Lin LL, Liu AJ, Liu JG, Yu XH, Qin LP, Su DF. Protective effects of scutellarin and breviscapine on brain and heart ischemia in rats. J Cardiovasc Pharmacol. 2007:50:327-32
32. Sun HD, Zhao QS. A drug for treating cardio-cerebrovascular diseasesphenolic compounds of Erigeron breviscapus. Prog Chem. 2009;21:77-83.

33. Li XL, Li YQ, Yan WM, Li HY, Xu H, Zheng XX, et al. A study of the cardioprotective effect of breviscapine during hypoxia of cardiomyocytes in vitro and during myocardial infarction in vivo. Planta Med. 2004;70:1039-44.

34. Tao YH, Jiang DY, Xu HB, Yang XL. Inhibitory effect of Erigeron breviscapus extract and its flavonoid components on GABA shunt enzymes. Phytomedicine. 2008;15:92-7.

35. Yang SC, Xu SZ, Wen GS, Liu YG, Xiao FH. Phenotypic diversity of populations in germplasm resources of Erigeron breviscapus. Acta Bot Boreal Occident Sin. 2008;28:1573-9.

36. Yang SC, Yang JW, Pan YH, Li GH, Liu BH. Comparison on agronomy and quality characters and breeding of new strains of Erigeron breviscapus. Zhongguo Zhong Yao Za Zhi. 2010;35:554-7.

37. Yang SC, Wang PL, Yang JW. Effects of genotypic and environmental on yield and scutellarin contentof Erigeron breviscapus. Chin Agric Sci Bull. 2011;27:140-3.

38. Li LY, Yang LY, Wang XG, Yang B, Yan SW, Li SP. Preliminary studies on breeding system and visiting insects of Erigeron breviscapus. Southwest China J Agric Sci. 2009;22:454-8.

39. Zhao WJ, Yan SQ, Cui MK, Zhang YF, Guan HL. The relationship between the stage of and rogenesis and flower character in Erigeron breviscapus. Lishizhen Med Mater Medica Res. 2010;21:1210-2.

40. Zhang SW, Ding F, He XH, Luo C, Huang GH. Characterization of the 'Xiangshui' lemon transcriptome by de novo assembly to discover genes associated with self-incompatibility. Mol Genet Genomics. 2014;290:365-75.

41. Zhou Q, Jia J, Huang X, Yan X, Cheng L, Chen S, et al. The large-scale investigation of gene expression in Leymus chinensis stigmas provides a valuable resource for understanding the mechanisms of poaceae selfincompatibility. BMC Genomics. 2014;15:399-413.

42. Stein JC, Howlett B, Boyes DC, Nasrallah ME, Nasrallah JB. Molecular cloning of a putative receptor protein kinase gene encoded at the selfincompatibility locus of Brassica oleracea. Proc Natl Acad Sci U S A. 1991;88:8816-20.

43. Hiscock SJ, McInnis SM. The diversity of self-incompatibility systems in flowering plants. Plant Biol. 2003;5:23-32.

44. Takayama S, Shiba H, Iwano M, Shimosato H, Che FS, Kai N, et al. The pollen determinant of self-incompatibility in Brassica campestris. Proc Natl Acad Sci U S A. 2000;97:1920-5.

45. Kemp BP, Doughty J. Scysteine-rich (SCR) biding domain analysis of the Brassica self-incompatibility S-locus receptor kinase. New Phytol. 2007;175:619-29.

46. Stone SL, Arnold M, Goring DR. A breakdown of Brassica self-incompatibility inARC1 antisense transgenic plants. Science. 1999;286:1729-31.

47. Kakita M, Murase K, Iwano M, Matsumoto T, Watanabe M, Shiba H, et al. Two distinct forms of M-locus protein kinase localize to the plasma membrane and interact directly with S-locus receptor kinase to transduce self-incompatibility signaling in Brassica rapa. Plant Cell. 2007;19:3961-73.

48. Samuel MA, Chong YT, Haasen KE, Aldea-Brydges MG, Stone SL, Goring DR. Cellular pathways regulating responses to compatible and selfincompatible pollen in Brassica and Arabidopsis stigmas intersect at Exo70A1, a putative component of the exocyst complex. Plant Cell. 2009:21:2655-71.

49. Bower MS, Matias DD, Fernandes CE, Mazzurco M, Gu T, Rothstein SJ, et al. Two members of the thioredoxin-h family interact with the kinase domain of a Brassica S locus receptor kinase. Plant Cell. 1996:8:1641-50.

50. Cabrillac D, Cock JM, Dumas C, Gaude T. The S-locus receptor kinase is inhibited by thioredoxins and activated by pollen coat proteins. Nature. 2001;410:220-3.

51. Vanoosthuyse V, Tichtinsky G, Dumas C, Gaude T, Cock JM. Interaction of calmodulin, a sorting nexin and kinase-associated protein phosphatase with the Brassica oleracea S-locus receptor kinase. Plant Physiol. 2003;133:919-29.

52. Yang B, Sugio A, White FF. Os8N3 is a host disease-susceptibility gene for bacterial blight of rice. Proc Natl Acad Sci U S A. 2006;103:10503-8.

53. Guan YF, Huang XY, Zhu J, Gao JF, Zhang HX, Yang ZN. RUPTURED POLLEN GRAIN1, a member of the MtN3/saliva gene family, is crucial for exine pattern formation and cell integrity of microspores in Arabidopsis. Plant Physiol. 2008;147:852-63. 
54. Hiscock SJ. Genetic control of self-incompatibility in Senecio squalidus L. (Asteraceae): a successful colonizing species. Heredity. 2000;85:10-9.

55. Haas BJ, Papanicolaou A, Yassour M, Grabherr M, Blood PD. De novo transcript sequence reconstruction from RNA-seq using the Trinity platform for reference generation and analysis. Nat Protoc. 2013;8:1494-512.

56. Pertea G, Huang X, Liang F, Antonescu V, Sultana R, Karamycheva S, et al. TIGR Gene Indices clustering tools (TGICL): a software system for fast clustering of large EST datasets. Bioinformatics. 2003;19:651-2.

57. Altschul SF, Madden TL, Schaffer AA, Zhang J, Zhang Z, Miller W, et al. Gapped BLAST and PSI-BLAST: a new generation of protein database search programs. Nucleic Acids Res. 1997;25:3389.

58. Deng $\mathrm{YY}, \mathrm{Li} J \mathrm{~J}, \mathrm{Wu} \mathrm{SF}$. Integrated $\mathrm{nr}$ database in protein annotation system and its localization. Comput Eng. 2006;32:71-4.

59. Apweiler R, Bairoch A, Wu CH. UniProt: the Universal Protein Knowledgebase [EB/OL]. Nucleic Acids Res. 2004. http:// nar.Oxfordjournals.org/cgi/content/full/32/supp1_1/d115, 32 (Database Issue):115.

60. Kanehisa M, Goto S, Kawashima S, Okuno Y, Hattori M. The KEGG resource for deciphering the genome. Nucleic Acids Res. 2004;32:D277-80.

61. Tatusov RL, Galperin MY, Natale DA, Koonin EV. The COG database: a tool for genome-scale analysis of protein functions and evolution. Nucleic Acids Res. 2000;28:33-6.

62. Li B, Dewey CN. RSEM: accurate transcript quantification from RNA-Seq data with or without a reference genome. BMC Bioinf. 2011;12:323.

63. Mortazavi A, Williams BA, McCue K, Schaeffer L, Wold B. Mapping and quantifying mammalian transcriptomes by RNA-Seq. Nat Methods. 2008;:5:621-8.

64. Robinson MD, McCarthy DJ, Smyth GK. edgeR: a Bioconductor package for differential expression analysis of digital gene expression data. Bioinformatics. 2010;26:139-40.

65. Anders S, Huber W. Differential expression analysis for sequence count data. Genome Boil. 2010;11:R106

\section{Submit your next manuscript to BioMed Central and take full advantage of:}

- Convenient online submission

- Thorough peer review

- No space constraints or color figure charges

- Immediate publication on acceptance

- Inclusion in PubMed, CAS, Scopus and Google Scholar

- Research which is freely available for redistribution 